\title{
"Seite an Seite": Ein Fallbericht über partnerschaftliche Unterstützung im Rahmen einer Brustkrebserkrankung
}

\author{
Tanja Zimmermann \\ Institut für Psychologie, Abteilung für Klinische Psychologie, Psychotherapie und Diagnostik, Technische Universität Braunschweig, \\ Deutschland
}

\section{Schlüsselwörter \\ Krebs · Psychologische Interventionen . \\ Psychoonkologie · Paartherapie}

\section{Zusammenfassung}

Krebserkrankungen, wie z.B. Brustkrebs, sind belastende Lebensereignisse, die erhebliche und andauernde Herausforderungen nicht nur für die erkrankte Frau, sondern auch für den Partner darstellen. Nach der Diagnosestellung treten häufig Ängste, Depressionen, sexuelle Störungen und Beziehungsstörungen auf. Die meisten psychoonkologischen Interventionsprogramme konzentrieren sich darauf, die betroffenen Frauen zu unterstützen und ihre individuellen Copingstrategien zu fördern. Jedoch sind auch die Partner durch die Krebserkrankung emotional belastet und stellen zudem für die betroffenen Frauen die wichtigste Quelle emotionaler und praktischer Unterstützung dar. Dennoch gibt es kaum Interventionen, die dies systematisch und standardisiert berücksichtigen.

Dieser Fallbericht beschreibt den Verlauf und die Ergebnisse eines psychoonkologischen Behandlungsprogramms für Paare («Seite an Seite»), dass dem Paar in 5 Sitzungen Informationen über die Krebserkrankung und -behandlung, Fertigkeiten zur Stress- und Krankheitsbewältigung, Kommunikationsfähigkeiten und dyadisches Coping sowie Hilfestellungen im Umgang mit Kindern und sexuellen Problemen vermittelt. Der Fallbericht zeigt beispielhaft, dass die partnerschaftliche Intervention, trotz massiver physischer Probleme und Beeinträchtigungen der individuellen Funktionsfähigkeit der Frau, zu einer gleichbleibend hohen Partnerschaftszufriedenheit bei beiden Partnern über den Zeitraum von über einem Jahr nach Diagnosestellung beiträgt.

\author{
Keywords \\ Cancer Psychological interventions . \\ Psycho-oncology · Couples therapy
}

\section{Summary}

'Side by Side': Couple-Based Psycho-Oncological Intervention Program for Women Diagnosed With

Breast Cancer: a Case Report

A cancer disease, such as breast cancer, is a stressful event in life that poses formidable and enduring challenges, not just to the women themselves, but also to their intimate partners. After the diagnosis anxiety disorders, depression, sexual and relationship problems may occur. Most of the psychosocial interventions focus on the women themselves and their individual coping strategies. However, partners are also highly affected by the cancer disease and, moreover, partners are considered as primary source of emotional and practical support for the women. There are few interventions for couples facing cancer, although both partners may be affected by the disease of one partner.

The present case report describes the procedure and the results of a psychosocial intervention for couples ('Side by Side'), which informs couples in 5 sessions about the disease and its medical treatment, individual and dyadic coping skills and provides support in communication and dealing with children and sexual problems. This case report demonstrates that a couple-based intervention leads, over one year after diagnosis, to a remaining high relationship satisfaction despite extreme physical problems and impact on the individual functioning of the woman.

\section{KARGER \\ Fax +497614520714 \\ Information@Karger.de}

www.karger.com (c) 2011 S. Karger GmbH, Freiburg

1016-6262/11/0213-0171\$38.00/0

Accessible online at:

www.karger.com/ver 


\section{Einleitung}

Neben kardiovaskulären Erkrankungen sind Krebserkrankungen die häufigsten chronischen Erkrankungen in Deutschland. Brustkrebs ist mit 58000 jährlichen Neuerkrankungen die häufigste Krebsneuerkrankung bei Frauen. Trotz seit 1980 steigender Inzidenz sinkt die Mortalität seit Mitte der 1990er Jahre leicht. Über alle Stadien der Krankheit betrachtet, liegt die relative 5-Jahres-Überlebensrate derzeit zwischen $83 \%$ und $87 \%$ [Robert Koch Institut (RKI), 2010]. Das mittlere Erkrankungsalter mit 64 Jahren liegt in einem Alter, in dem die meisten Frauen (etwa 63\%) in einer Partnerschaft leben. Die Diagnose und medizinische Behandlung einer Brustkrebserkrankung stellt in den meisten Fällen einen massiven Stressor für die Patientin dar und beeinflusst auch das Wohlbefinden des Partners und der Familie.

Eine Vielzahl von psychologischen und psychosozialen Interventionen wurde in den letzten Jahrzehnten als ergänzende Verfahren zum medizinischen Vorgehen entwickelt. Meta-Analysen deuten mit wenigen Ausnahmen auf eine positive globale Wirkung bei Krebserkrankungen hin [Martire et al., 2004; Osborn et al., 2006; Zimmermann und Heinrichs, 2006; Zimmermann et al., 2007]. Die Ergebnisse bestätigen, dass es bereits etablierte hilfreiche Interventionsformen für gynäkologische Krebspatienten gibt, die jedoch in ihrem Ausmaß im Durchschnitt nicht über einen kleinen bis mittleren positiven Effekt hinaus kommen. Eine Möglichkeit die Wirksamkeit zu optimieren, könnte darin liegen, die Intervention stärker auf krankheitsspezifische Aspekte maßzuschneiden. Sexualität und Körperbild sind Bereiche, die durch eine Brustkrebserkrankung massiv beeinträchtigt werden und auch nachhaltige Auswirkungen auf die Partnerschaft haben können. Interventionen, die diese Aspekte berücksichtigen, zeigen deutliche Verbesserungen bei den Patientinnen in den Bereichen Depression, Angst, Körperbild, Partnerschaftszufriedenheit, Orgasmushäufigkeit und Kommunikation über sexuelle Wünsche [Kalaitzi et al., 2007].

Partnerschaftlich fokussierte Interventionen, die relevante partnerschaftliche Prozesse identifizieren und beinhalten, können zu einer besseren Anpassung an die Krebserkrankung für beide Partner in einer Beziehung führen [Manne und Badr, 2008]. In einem aktuellen Review über paarorientierte Interventionen für chronisch Kranke zeigte sich eine Überlegenheit dieser Interventionen in Bezug auf Depressivität des Patienten, partnerschaftliche Funktionsfähigkeit und Schmerz im Vergleich zu patientenorientierten Angeboten oder «treatment-as-usual» [Martire et al., 2010]. Die Einbeziehung des Partners um nicht nur der Patientin bei der Bewältigung der Krebserkrankung zu helfen, sondern auch den Partner zu unterstützen, die Partnerschaft zu stärken und Verschlechterungen der interpersonellen Beziehung vorzubeugen, sind die Ziele des psychoonkologischen Behandlungsprogramms «Seite an Seite» [Heinrichs und Zimmermann, 2008; Zimmermann und Heinrichs, 2008].

\section{Überblick über die Behandlung}

Das «Seite an Seite»-Programm richtet sich an Paare, bei denen die Frau an Brustkrebs oder gynäkologischem Krebs in Stadium I-III erkrankt ist. Die Intervention besteht aus 5 Sitzungen (jede etwa 1,5-2 h), die in einem Abstand von 2-3 Wochen begleitend zu der akuten medizinischen Behandlung stattfinden. Die fünfte Sitzung (Booster-Sitzung) erfolgt etwa 6 Monate später. Alle Sitzungen werden mit der Patientin und dem Partner gemeinsam durchgeführt.

Das Programm beinhaltet Informationen über die Krebserkrankung und ihre Behandlung, setzt die Diagnose mit einem stressauslösenden Ereignis gleich und bietet dementsprechend Fertigkeiten zur Stressbewältigung an (Tab. 1). Bedeutsam ist jedoch, dass es sich überwiegend um dyadischsupportive Skills handelt, wie z.B. die Anleitung über krebsrelevante Inhalte miteinander zu sprechen, die eigene Belastung zu vermitteln, Unterstützung des Partners/der Partnerin anzunehmen und Rückmeldung darüber zu geben, wie diese Unterstützung wahrgenommen wurde. Darüber hinaus geht es um die Frage, wie man Intimität in der Partnerschaft (neu) erleben kann, nachdem man eine Vielzahl von körperlichen Herausforderungen und Veränderungen erlebt hat.

Die Ergebnisse einer randomisiert kontrollierten Studie mit 72 Paaren [Heinrichs et al., persönliche Mitteilung] weisen darauf hin, dass Frauen, die mit ihrem Partner an «Seite an Seite» teilgenommen haben, nach der Intervention eine größere Reduktion der krebsspezifischen Belastungen und Progredienzangst zeigten als Patientinnen in der Kontrollgruppe. Darüber hinaus fanden sich bei Paaren in der «Seite an Seite»-Bedingung weniger Vermeidung in der Auseinandersetzung mit der Krebserkrankung, mehr posttraumatische Reifung und bessere Kommunikationsfertigkeiten sowie dyadisches Coping (DC). Trotz andauernder medizinischer Behandlung zeigte sich eine Zunahme sexueller Aktivitäten [Zimmermann und Heinrichs, 2011]. Anhand einer Kasuistik soll die Vorgehensweise im Rahmen dieses Interventionsprogramms vorgestellt werden. Es werden die wichtigsten Aspekte jeder Sitzung dargestellt.

\section{Fallbericht}

Eine 40-jährige Patientin meldete sich mit ihrem 41-jährigen Ehemann an. Die Partnerschaft bestand seit 12 Jahren; das Paar hatte keine Kinder. Beide hatten einen Realschulabschluss. Die Patientin arbeitete als Arzthelferin (zur Zeit krankgeschrieben); der Ehemann war selbstständig. Zum Zeitpunkt der 1. Sitzung und Prä-Messung lag die Diagnose Brustkrebs der Patientin knapp 2 Monate zurück. Die Patientin war in Stadium I $\left(\mathrm{T}_{1} \mathrm{~N}_{0} \mathrm{M}_{0}\right)$ erkrankt. Eine brusterhaltende Operation vor etwa einem Monat sowie die erste Chemotherapie, von insgesamt 6, war vor 10 Tagen erfolgt.

Sitzung 1: Psychoedukation über die Erkrankung, ihrer Auswirkung auf Körper und Seele sowie Einführung in die partnerschaftliche Unterstïtzung Psychoedukation: Zu Beginn der Sitzung wurde mit dem Paar die Zeit der Diagnosestellung reflektiert und erörtert wie jeder Einzelne die Dia- 
Tabelle 1. Inhaltlicher Überblick über die 5 «Seite an Seite»-Sitzungen

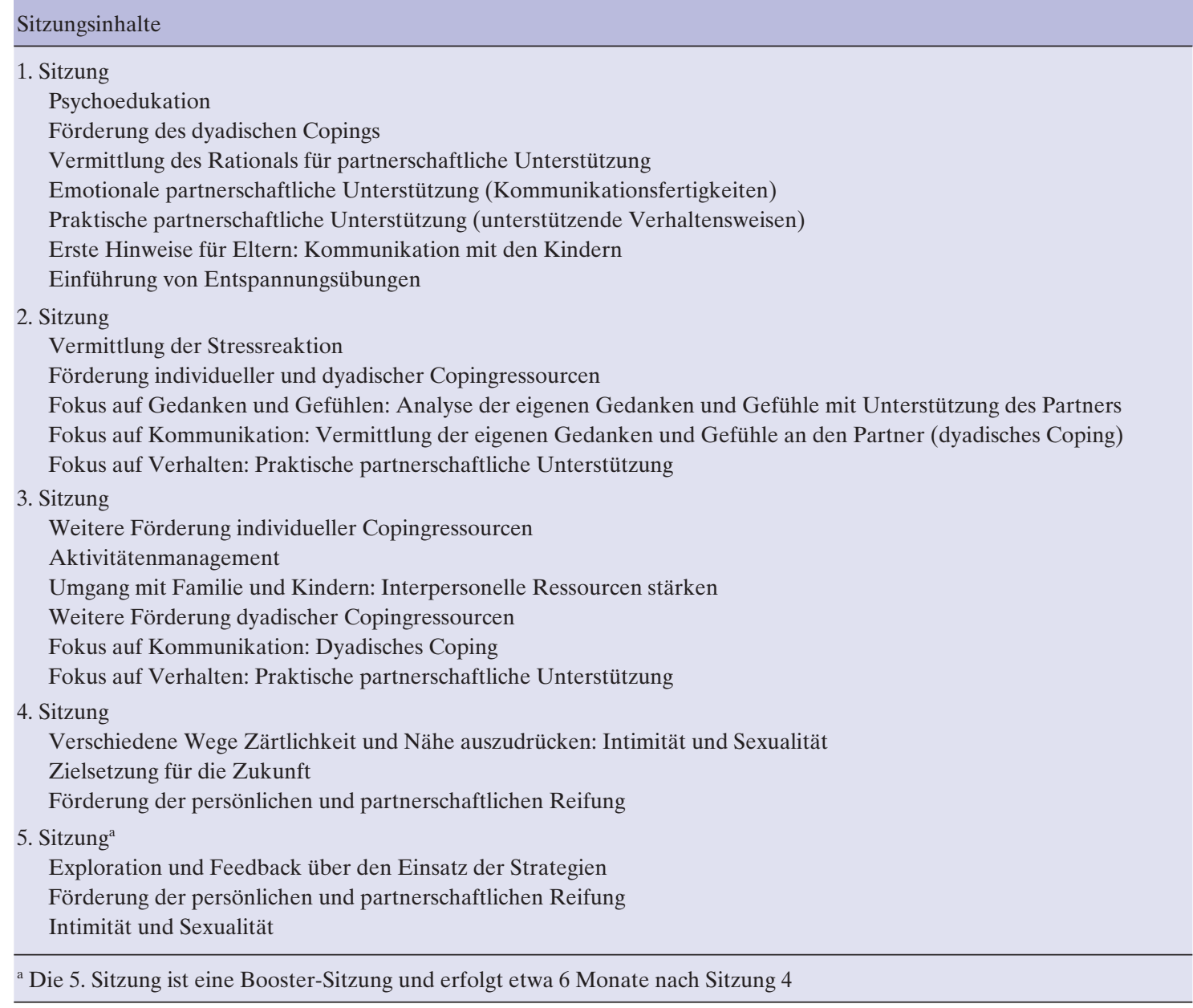

gnose und die medizinischen Behandlungsempfehlungen verstanden hat Die Patientin berichtete von einer Routineuntersuchung beim Gynäkologen, bei der ein Knoten in der Brust entdeckt wurde sowie einer nachfolgenden Mammografie und Stanzbiopsie mit zunächst unklarem Ergebnis. Nach etwa 2 Wochen stand die Diagnose Brustkrebs fest. Beide Partner fühlten sich durch die Diagnose und anfängliche Ungewissheit stark belastet. Da beide bereits Erfahrungen mit Krebserkrankungen gemacht hatten (bei beiden Partnern verstarben die Mütter durch Brustkrebserkrankungen, zudem waren eine Tante, eine Cousine und die Chefin der Patientin sowie Freunde des Mannes an Krebs erkrankt), wurde geklärt, ob diese negativen und stark angstassoziierten Erfahrungen mit Krebs die gegenwärtigen Erwartungen und Meinungen über die Prognose und Behandlung der Frau ungünstig beeinflussten. Es stellte sich heraus, dass die Patientin Parallelen zur Erkrankung ihrer Mutter sah, aber auch Unterschiede (z.B. das Stadium der Erkrankung) herausarbeiten konnte. Um eine genetische Komponente festzustellen, war eine humangenetische Untersuchung geplant. Der Partner hingegen sah keine Parallelen der Erkrankung seiner Frau zu seinen bisherigen Erfahrungen mit Krebs. Beide waren recht gut über die Diagnose und medizinische Behandlung informiert, so dass eine weitere Besprechung nicht notwendig war. Der Fokus lag hier vor allem auf der Vermittlung psychischer Reaktionen auf die Krebserkrankung und nicht auf der Vermittlung medizinischer Befunde. Dies ist Aufgabe (und Expertise) des Arztes und sollte nicht Teil psychoonkologischer Unterstützung sein, sofern das betroffene Paar ein angemessenes Verständnis der Erkrankung entwickelt hat.

Die Operation der Brust erfolgte brusterhaltend. Als weitere medizinische Schritte waren Chemo-, Strahlen- und Hormontherapie geplant. Im Folgenden wurden die Sorgen des Paares exploriert und unrealisti- sche, irrationale Gedanken hinterfragt. Die Patientin machte sich große Sorgen bezüglich des bevorstehenden Haarverlusts durch die bereits begonnene Chemotherapie. Zum einen hatte sie Angst vor ihrem veränderten Erscheinungsbild, zum anderen machte sie sich auch Sorgen wie ihr Mann auf ihre Glatze reagieren würde. Auch Gedanken, dass dann «alle sehen, dass ich krank bin» gingen ihr durch den Kopf. Der Ehemann hingegen reagierte auf die Sorgen der Frau eher pragmatisch («die Haare wachsen ja wieder»). Es wurde deutlich, dass sich das Paar in ihren Verarbeitungs- und Copingstrategien unterschied. Während die Patientin vorrangig emotional reagierte, stand bei ihrem Partner eine problemorientierte, wenig emotionale Herangehensweise im Vordergrund.

Förderung des DC: Eine erste Übung befasste sich mit der emotionalen Reaktion auf die Diagnose jedes einzelnen Partners, indem beide (nacheinander) gebeten wurden, ihre ersten Reaktionen zu berichten. Der andere Partner hörte währenddessen zu, um hinterher das Gesagte zusammenzufassen. Wie zu erwarten konnte die Patientin ihre Emotionen in Bezug auf die Diagnose und Behandlung recht gut verbalisieren, auch war ihr Partner in der Lage, diese dann zusammenzufassen. Der Partner hingegen hatte erhebliche Schwierigkeiten, seine Gefühle zu äuBern. In der weiteren Exploration durch die Therapeutin wurde deutlich, dass er seine Frau nicht zusätzlich belasten wollte indem er von seinen Ängsten und Sorgen in Bezug auf ihre Erkrankung sprach («protective buffering»). Die Patientin berichtete im Anschluss an die Übung von Unzufriedenheiten mit der partnerschaftlichen Kommunikation, die sie jedoch zum gegenwärtigen Zeitpunkt nicht näher spezifizieren konnte. Da bereits zu diesem Zeitpunkt deutliche Unterschiede im Ausmaß der emotionalen und praktischen Unterstützung beider Partner erkennbar wurden, konnte daran sehr gut das Rational für die Relevanz partner- 
schaftlicher Unterstützung vermittelt werden. Eine häufige Reaktion von Partnern erkrankter Frauen ist ein Gefühl der Hilflosigkeit, da sie nicht wissen, wie sie ihre Frauen am besten unterstützen können und besorgt sind das Falsche zu sagen oder zu tun. Der Ehemann fand sich in dieser Beschreibung wieder.

Dem Paar wurde verdeutlicht, dass «Seite an Seite» beiden Partner helfen soll Wege kennen zu lernen, sich gegenseitig effektive emotionale und praktische Unterstützung zukommen zu lassen, mit dem Ziel des Stressabbaus bei beiden Partnern und einer weiteren Kohäsion des Paares. Auch die vermittelten Unterschiede in den Kernfähigkeiten von Mann und Frau waren für das Paar eingängig. Wie andere Männer häufig auch, zeigte der Partner eine stärkere Ausprägung der praktischen im Vergleich zur emotionalen Unterstützung, wohingegen die Patientin bereits recht gut in der emotionalen Unterstützung war, aber selten Rückmeldung an den Partner gab, was sie sich wünschte oder was ihr gut oder auch nicht so gut gefiel.

Im Anschluss wurde die Bedeutung unterstützender Kommunikation, insbesondere für die emotionale Unterstützung, mit dem Paar erörtert und die Sprecher- und Zuhörerregeln vermittelt. Beide konnten diese gut annehmen und fanden Parallelen zu Kommunikationsregeln, die sie schon im beruflichen Kontext erfahren hatten. Beide konnten auch klar identifizieren, welche Regeln sie schon gut umsetzen konnten und bei welchen sie noch Schwierigkeiten hatten. Der Patientin gelang das Sichöffnen und das aufnehmende Zuhören schon gut. Probleme identifizierte sie bei Ich-Gebrauch, Konkretheit und Zusammenfassen. Bei der Reflektion ihres Kommunikationsverhaltens gegenüber ihrem Mann fiel ihr auf, dass sie häufig vorwurfsvoll war und erwartete, dass er bestimmte Dinge von sich aus wisse, ohne dass sie diese explizit sagte. Der Partner konnte für sich feststellen, dass er Schwierigkeiten mit dem Sich-öffnen hatte, aber auch nicht zusammenfasste und nicht konkret nachfragte. Er versuchte oft aus bestimmten Gesten oder Verhaltensweisen seiner Frau «abzulesen» wie sie sich fühlte oder was in ihr vorging. Beiden leuchtete ein, dass «Gedankenlesen» wenig erfolgreich ist.

Praktische partnerschaftliche Unterstützung: Um mit dem Paar die praktische Unterstützung zu spezifizieren, wurden die «Füreinander-Listen» mit einer Auswahl von unterstützenden Verhaltensweisen verwendet. Die Patientin wählte 5 Dinge aus, die sie sich von ihrem Mann bis zur nächsten Sitzung wünschte: Um Sex bitten, etwas Angenehmes gemeinsam unternehmen (z.B. spazieren gehen), mit der Patientin über seine Ängste und Sorgen bezüglich der Erkrankung sprechen, der Patientin Zeit für sich alleine geben und sie liebevoll in den Arm nehmen. Der Partner wählte 2 Dinge aus seiner Liste: Mit ihm kuscheln oder schmusen und ihn in erotischer Weise berühren.

\section{Sitzung 2: Krebsbewältigungstraining I - Emotionale und praktische} Unterstützung der Partner festigen

$\mathrm{Zu}$ Beginn der Sitzung war eine angespannte Stimmung zwischen dem Paar spürbar. Bei genauerer Exploration stellte sich heraus, dass seit einigen Tagen schlechte Stimmung herrschte und nur das Nötigste gesprochen wurde. Die Patientin berichtete, dass ihr Mann an dem Abend an dem sie ihre zweite Chemotherapie erhielt, beruflich unterwegs war und sie ihn telefonisch nicht erreichen konnte. Sie war verärgert und machte ihm Vorwürfe. Die Vorkommnisse der letzten Tage wurden genutzt, um das Gelernte der letzten Sitzung darauf anzuwenden: Die Kommunikationsregeln wurden wiederholt und beide erhielten die Möglichkeit, dieses Ereignis unter Anleitung der Therapeutin gemeinsam zu besprechen.

Vermittlung der Stressreaktion: Für beide Partner wurden die einzelnen Komponenten der Stressreaktion (Gedanken, Gefühle, Verhalten und körperliche Veränderungen) identifiziert. Es zeigte sich, dass beide bei der Formulierung der Gedanken Unterstützung benötigten und es ihnen schwer fiel, Gefühle von Gedanken zu trennen. Zudem berichtete die Patientin von belastenden Gedanken und damit zusammenhängender negativer Stimmung. Die kognitive Komponente der Stressreaktion wurde vertieft. Dabei waren für die Patientin die «Bewältigenden Selbst- gespräche» (Spaltenschema) sehr hilfreich. Sie konnte eigene dysfunktionale Kognitionen identifizieren und dann, mit Unterstützung durch den Partner, kognitive Fehler erkennen und bewältigende Gedanken entwickeln. Beispielsweise hatte sie nach der ersten Chemotherapie bezüglich des Haarverlustes Katastrophisierungen («das wird alles ganz fürchterlich») und zog voreilige Schlüsse («ich werde so hässlich aussehen»). In der Zwischenzeit hatte sie ihre Haare verloren. Die Lösung ein Tuch zu tragen, gefiel ihr sehr gut und sie fühlte sich damit auch wohl. Der Patientin wurde deutlich, dass die Gedanken im Vorfeld wenig hilfreich waren und sie deutlich belasteten.

Förderung dyadischer Copingressourcen: Mit dem Paar wurde erarbeitet, dass sich Partner sehr stark in der Wahrnehmung von Stress unterscheiden können und dementsprechend der eine Partner den Stress und das Stresserleben des anderen auch nicht vorhersehen (hellsehen) kann. Als aktuelles Beispiel wurde die Eingangssituation verwendet, bei der dem Mann nicht klar war, dass seine Frau ihn telefonisch erreichen wollte und vor allem wie wichtig ihr das war. Für eine gegenseitige hilfreiche Unterstützung ist es bedeutsam, den Stress des anderen nachfühlen zu können (ohne zwingend genauso zu fühlen). Mit Hilfe der «Trichtermethode» von Bodenmann [2004] ist eine emotionale Aktivierung beider Partner möglich, um sich dann gegenseitig angemessen unterstützen zu können. Das Ziel des DC besteht darin, beide durch den Aufbau von Intimität dazu zu ermutigen, sich dem Partner zu öffnen - in einem schützenden Rahmen, den die Therapeutin gewährleistet. Die Frau wählte in der Sprecherrolle das Thema einer möglichen operativen Entfernung der Eierstöcke zur Vorbeugung weiterer Krebserkrankungen. Nachdem sie zunächst die Stresssituation beschrieb, konnte in dieser ersten Phase des DC relativ schnell durch gezieltes Fragen der Therapeutin eine Aktivierung des emotionalen Erlebens erreicht werden. Emotionen und Gedanken wurden gesammelt und dann ein Gefühl ausgewählt (Angst), um auf tiefer liegende Gefühle (Scham) und Kognitionen («ohne Brust und Eierstöcke bin ich keine Frau mehr» und «ich bin nicht mehr liebenswert») zu fokussieren und das semantische Netzwerk zu aktivieren. Durch mehrfache Aufforderung an den Ehemann, immer wieder zusammenzufassen, insbesondere auch die geäußerten Emotionen, gelang eine emotionale Aktivierung und Betroffenheit auch bei ihm, so dass in Phase 2 übergeleitet werden konnte. In dieser Phase soll der Zuhörer (Mann) den Sprecher unterstützen. Durch die emotionale Aktivierung des Partners war es möglich, dass dieser auf derselben Ebene Unterstützung liefern konnte, auf der auch der Stress geäußert wurde (in diesem Fall auf der emotionalen Ebene). Der Mann äußerte gegenüber der Frau, dass er ihren Wert nicht an Äußerlichkeiten festmacht und beschrieb eine Reihe von Persönlichkeitseigenschaften, die er an seiner Frau liebt, um sie anschließend auch in den Arm zu nehmen. In Phase 3 meldete die Frau ihrem Mann dann zurück, wie die Unterstützung bei ihr ankam (sie war zu Tränen gerührt und empfand die Äußerungen des Mannes als sehr hilfreich und beruhigend). Beide Partner äußerten nach dem Rollenspiel bisher noch nie so intensiv über ein Thema gesprochen zu haben. Der Mann war überrascht über die Ängste und Sorgen seiner Frau, aber auch erleichtert, dass sie so offen geäußert wurden und er somit auch in der Lage war seine Frau konkret zu unterstützen. Ein anschließend angestrebter Rollentausch erwies sich als schwierig. Wie schon in Sitzung 1 deutlich wurde, hatte der Partner massive Schwierigkeiten, sich zu öffnen und seine Ängste und Sorgen in Bezug auf die Erkrankung seiner Frau anzusprechen. Es wurde dann ein Thema gewählt, das nicht direkt mit der Krebserkrankung zusammenhing. Es ging darum, dass seine Frau einen Ring, den er ihr geschenkt hatte, gegen einen anderen Ring, den sie von ihrer Freundin geschenkt bekommen hatte, ausgetauscht hatte. In den 3 Phasen des DC konnten seine Gefühle (Enttäuschung, verletzt sein) und Gedanken («sie liebt mich nicht mehr so, weil sie meinen Ring abgelegt hat») gut herausgearbeitet werden, so dass die Frau auch emotional aktiviert wurde und entsprechende Unterstützung anbieten konnte. Die Frau äußerte nach dem Rollenspiel, dass ihr vorher gar nicht klar war, wie bedeutsam das Tragen des Ringes für ihren Mann war und sie froh war, dass er dies offen ansprechen konnte. 
Sitzung 3: Krebsbewältigungstraining II - Integration der Erkrankung in das gegenwärtige Leben

Weitere Förderung dyadischer Copingressourcen: Die Fertigkeiten zum DC wurden weiter vertieft, indem die 3 Phasen-Methode aus Sitzung 2 erneut durchgeführt wurde. Beide Partner berichteten von deutlichen Verbesserungen im Kommunikationsverhalten - auch zwischen den Sitzungen. Die letzte Sitzung hatte «aufgerüttelt», z.B. überlegte die Frau mehr, wie sie ihrem Mann etwas sagt (weniger vorwurfsvoll, mehr Ich-Botschaften). Der Ehemann berichtete, dass es ihm durch die Offenheit seiner Frau leichter fiel sie zu unterstützen und er sich somit weniger hilflos fühlte.

Aktivitätenmanagement: Aktivitätenmanagement wurde als eine Form der Stressbewältigung und zur Steigerung der Lebensqualität eingeführt. Gemeinsam wurde diskutiert, inwieweit die Krebserkrankung zu Unterbrechungen in der normalen Routine des Paares geführt hat und auch zum Wegfall angenehmer Aktivitäten. Die Relevanz positiver Aktivitäten wurde unter Zuhilfenahme des erarbeiteten Stressmodels aus Sitzung 2 betont. Mit dem Paar wurde eine Brainstorming-Liste mit individuellen (Patientin: Spinning, Schwimmen; Partner: Angeln) und dyadischen Aktivitäten (joggen, Langlauf, Inliner oder Rad fahren, spazieren gehen) erstellt. Anschließend wurde überlegt, welche Aktivitäten auch zum Zeitpunkt der Chemotherapie möglich wären und das Paar wurde ermutigt, diese auch durchzuführen.

Umgang mit Familie und Kindern: Interpersonelle Ressourcen sollten gestärkt werden. Da das Paar keine Kinder hatte, wurde exploriert ob es Probleme mit anderen Familienangehörigen oder Freunden/Bekannten in Bezug auf die Erkrankung gab. Beide fühlten sich gut durch Freunde unterstützt und waren auch in der Lage offen über die Erkrankung zu sprechen. Die Patientin berichtete allerdings von Schwierigkeiten mit ihrer Chefin, die selber auch an Krebs erkrankt war. Sie sei wenig verständnisvoll und fordernd. Die Patientin fühlte sich in Bezug auf Fragen nach der Wiederaufnahme ihrer Arbeit unter Druck gesetzt und überfordert. Diese Emotionen konnten im Gespräch exploriert werden und gemeinsam mit dem Mann wurden Strategien zum zukünftigen Umgang mit der Chefin überlegt. Die Patientin empfand es als entlastend zu wissen, dass sie gegenüber der Chefin nicht verpflichtet war, detailliert Auskunft über ihre Erkrankung und Behandlung geben zu müssen. Auch die vom Mann angebotene Unterstützung, sie beim nächsten Gespräch mit der Chefin zu begleiten, führte zu einer Entlastung.

Sitzung 4: Krebsbewältigungstraining III - Integration der Erkrankung in das zukünftige Leben

Das Paar erlebte weiterhin Verbesserungen in der Kommunikation zwischen den Sitzungen. Beide achteten verstärkt auf die Regeln, wodurch insbesondere Vorwürfe deutlich reduziert wurden. Die in der 3. Sitzung geplanten Aktivitäten konnten teilweise ausgeführt werden (z.B. spazieren gehen), mussten aber aufgrund massiver Nebenwirkungen nach der 4. Chemotherapie eingeschränkt werden.

Erste Förderung der persönlichen und partnerschaftlichen Reifung und Zielsetzung für die Zukunft: Mit dem Paar wurde besprochen, dass die Konfrontation mit einer lebensbedrohlichen Erkrankung dazu führen kann, die Grundannahmen oder bisherigen Lebensziele einer Person in Frage zu stellen, aber auch dazu, positive Aspekte dieser Erfahrung zu erkennen. Beiden fiel es schwer zum damaligen Zeitpunkt positive Aspekte zu identifizieren. Rückblickend konnte dem Paar vermittelt werden was sie in den vergangenen Wochen geleistet hatten. Beide führten an, dass sie bereits in der Vergangenheit einige schwierige Situationen (z.B. unerfüllten Kinderwunsch) gut gemeinsam gemeistert hatten und somit zuversichtlich seien, auch diese Krise zu überstehen.

Eine graduelle Zielsetzungsaufgabe mit individuellen und partnerschaftlichen sowie kurz- und langfristigen Zielen zeigte, dass die Patientin schnell in der Lage war, sowohl individuelle (z.B. Sport, weniger arbeiten) als auch dyadische Ziele (z.B. sportliche Aktivitäten, Kreuzfahrt) zu generieren. Ihrem Mann fiel es insbesondere schwer individuelle Ziele festzulegen. Bei weiterer Exploration zeigte sich, dass er seine Ziele und Aktivitäten in erster Linie auf die Partnerschaft ausgerichtet hatte, so dass wenig Raum für Individuelles blieb. Er konnte mit Hilfe der Frau ermuntert werden sich auch eigene Ziele zu setzen.

Sexualität: Bereits zu Beginn der Sitzungen wurde deutlich, dass Sexualität ein wichtiger Bestandteil der Partnerschaft war, da das Thema immer wieder aufkam (z.B. bei den Wünschen in der Füreinander-Liste aus Sitzung 1). Bei der Exploration der gegenwärtigen Sexualität zeigte sich, dass es schon vor der Erkrankung sexuelle Probleme gab und seit Diagnosestellung kein Geschlechtsverkehr mehr stattfand. Anhand einer Informationsbroschüre wurden potenzielle Nebenwirkungen der aktuellen Behandlung (z.B. eingeschränkte Lubrikationsfähigkeit durch Chemotherapie) sowie Interventionsmöglichkeiten besprochen.

Mit Hilfe der Übungskarten zur sexuellen Kommunikation, bei denen beide Partner unabhängig voneinander ein Haus aus Begriffen legten, die ihnen im Hinblick auf die eigene Sexualität wichtig waren, konnte ein offenes Gespräch über die aktuelle und vergangene Sexualität des Paares angeregt werden. Beide erklärten dem anderen ihr «Haus», wodurch die sexuelle Kommunikation vertieft wurde. Die Patientin berichtete über negative sexuelle Erfahrungen im Kindesalter und die damit zusammenhängenden Probleme sich fallen zu lassen. Hinzu kamen Probleme mit dem Körperbild. Sie beschrieb, sich weniger attraktiv, weiblich und sexy zu fühlen und Berührungen an der operierten Brust - auch aufgrund von Taubheitsgefühlen - als unangenehm zu erleben. Dennoch äußerte sie den Wunsch weiterhin sexuell aktiv sein zu wollen, bat ihren Partner jedoch um Geduld und darum, die operierte Brust zunächst nicht zu berühren. Auch äußerte sie den Wunsch, mit ihrem Mann abends einfach nur kuscheln zu wollen, ohne dass es zum Geschlechtsverkehr kommen müsse. Der Ehemann beschrieb in seinem «Haus» die für ihn wichtigen Begriffe in Bezug auf Sexualität. Dabei bewertete er die gemeinsame Kommunikation als Fundament, auf dem Lust und Humor, aber auch Vertrauen, Verständnis, Begehren und Glück aufbauten. In seiner Beschreibung machte er deutlich, dass er seine Frau nach wie vor sehr attraktiv fand (auch die operierte Brust) und sich Körperkontakt (wie schmusen und auch Geschlechtsverkehr) wünschte. Die Patientin war nach den Ausführungen des Mannes zu Tränen gerührt. Es zeigten sich viele Gemeinsamkeiten zwischen den Häusern in Bezug auf die verwendeten Begriffe, aber auch im Hinblick auf deren Wichtigkeit und somit Anordnung im Haus. Die Übung führte zu einem offenen Gespräch, bei dem beide offen ihre Ängste, Sorgen und Wünsche äußern konnten.

\section{Sitzung 5 (Booster): Persönliche und partnerschaftliche Reifung}

Diese Sitzung fand 6 Monate nach Sitzung 4 statt. Die Patientin hatte in der Zwischenzeit die Chemotherapie abgeschlossen. Die humangenetische Untersuchung belegte einen Gendefekt, so dass in einer erneuten Operation die andere Brust und die Eierstöcke entfernt werden sollten. Eine Strahlentherapie war somit nicht mehr erforderlich.

Ziel dieser Sitzung war die Besprechung des Fortschritts dyadischer Bewältigungsstrategien, der sexuellen Funktionsfähigkeit und die Umsetzung der Zielsetzungsaufgabe sowie die Förderung der persönlichen und partnerschaftlichen Reifung. Die Patientin fühlte sich nach wie vor gut von ihrem Mann unterstützt. Beide berichteten, dass sie gemeinsam als Paar gut zurecht kämen. Belastende und dysfunktionale Gedanken, die aufkamen, konnten sie gut mit den erlernten Strategien bearbeiten. Es zeigte sich, dass der Bereich Sexualität weiterhin problematisch war. Es gab zwar in der Zwischenzeit Geschlechtsverkehr, allerdings litt die Patientin unter einer deutlichen Einschränkung der sexuellen Appetenz. Im Gespräch zeigte sich, dass das Paar jedoch in der Lage war über die Probleme zu sprechen und die damit zusammenhängenden Ängste und Sorgen zu thematisieren.

\section{Schlussfolgerungen}

Die Fallbeschreibung verdeutlicht wie vielschichtig die individuellen und dyadischen Reaktionen auf eine Brustkrebser- 
Abb. 1. Individuelle Funktionsfähigkeit des Paares im Zusammenhang mit der medizinischen Behandlung der Frau über 4 Messzeitpunkte und im Vergleich zu allen Paaren der Kontrollbedingung der «Seite an Seite»-Studie ( $\mathrm{N}=34$; Heinrichs et al., persönliche Mitteilung; Prä-Intervention: Vor
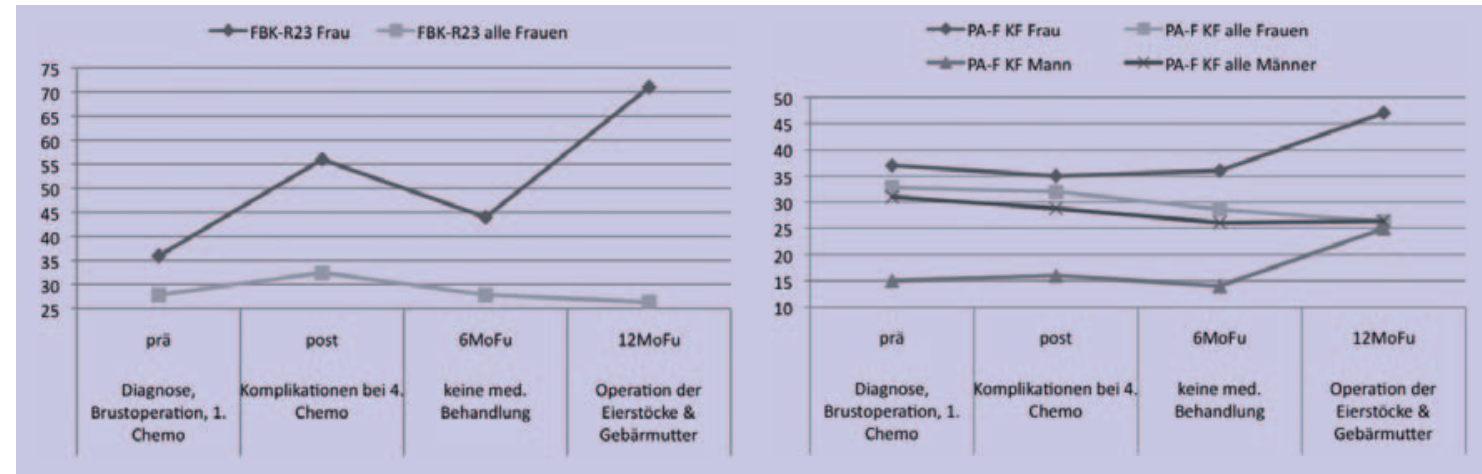

Sitzung 1; Post-Intervention: Nach Sitzung 4; 6MoFU: 6 Monate nach post; 12MoFU: 12 Monate nach post; FBK-R23: Fragebogen zur Belastung einer Krebserkrankung, range 0-115, cut off >34; PA-F KF: Progredienzangstfragebogen Kurzform, range 12-60, cut off >34).

Abb. 2. Dyadische Funktionsfähigkeit des Paares im Zusammenhang mit der medizinischen Behandlung der Frau über 4 Messzeitpunkte und im Vergleich zu allen Paaren der Kontrollbedingung der «Seite an Seite»-Studie $(\mathrm{N}=34$; Heinrichs et al., persönliche Mitteilung; Prä-Intervention: Vor
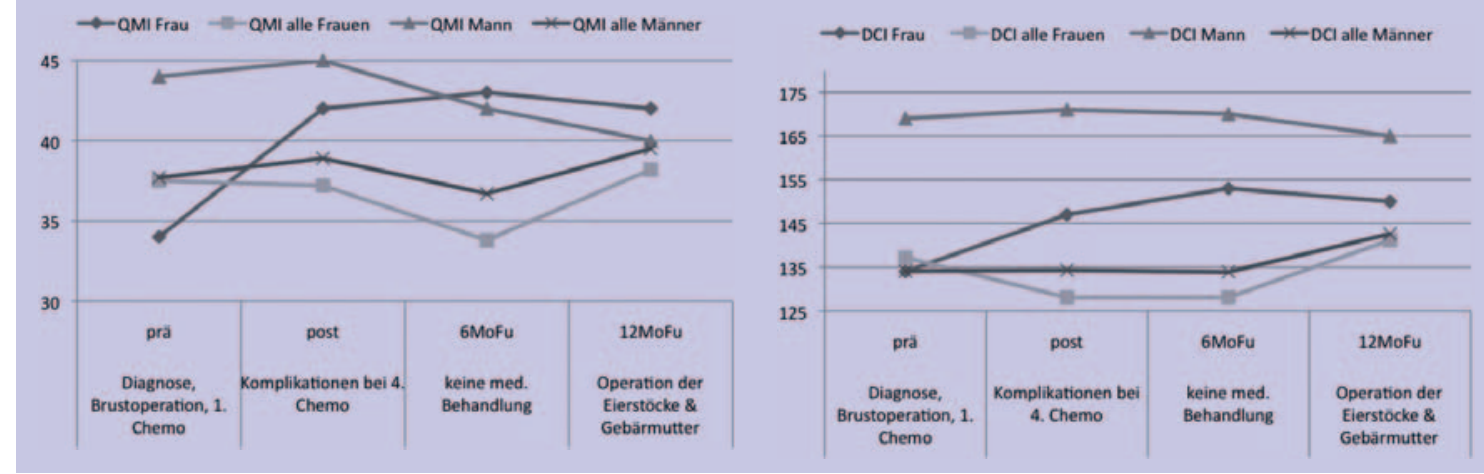

Sitzung 1; Post-Intervention: Nach Sitzung 4; 6MoFU: 6 Monate nach post; 12MoFU: 12 Monate nach post; QMI: Quality of Marriage Index, range 6-45; DCI: Dyadisches Coping Inventar, range 35-175; <111 geringes dyadisches Coping, 111-145 moderates dyadisches Coping,

$>145$ hohes dyadisches Coping).

krankung der Frau sein können. Die Ergebnisse der Fragebogenuntersuchung zeigen deutliche Unterschiede zwischen den Partnern in der individuellen Belastung (Abb. 1). Weist die Frau eine ausgeprägte psychische Belastung durch die Krebserkrankung (Fragebogen zur Belastung einer Krebserkrankung, FBK-R23), verbunden mit Progredienzangst (Progredienzangstfragebogen Kurzform, PA-F KF) über alle Messzeitpunkte auf, finden sich beim Partner unauffällige Werte.

Die Ergebnisse des Paares werden im Folgenden in Bezug gesetzt zu den Ergebnissen der Paare, die im Rahmen der randomisiert kontrollierten Studie teilnahmen und keine Intervention erhielten $(\mathrm{N}=34)$ [Heinrichs et al., persönliche Mitteilung]. Im Vergleich zu den anderen Frauen der Gesamtstichprobe war die Patientin zu allen Messzeitpunkten deutlich belasteter als die Frauen insgesamt. Setzt man die Werte allerdings in Bezug zu ihrer individuellen medizinischen Behandlung, lassen sich somatische Belastungen identifizieren, die sich vermutlich auch in den Messwerten abbilden (Anstieg der Belastung zum post-Zeitpunkt und 12 Monate nach post $(12 \mathrm{MoFu}))$. So gab es zum post-Zeitpunkt starke Komplikationen bei der 4 . Chemotherapie und vor dem $12 \mathrm{MoFu}$ fand eine Unterleibsoperation statt. Die Progredienzangst der Frau ist zum prä- und post-Zeitpunkt vergleichbar mit der Angst der Frauen insgesamt. Zum 12MoFu (und Zeitpunkt der Eierstock- und Gebärmutteroperation) zeigt sich eine Zunahme der Angst. Die Werte des Partners zeigen im Vergleich zu den Männern insgesamt niedrigere Werte in Bezug auf Progredienzangst. Allerdings lässt sich auch hier - wie bei der Frau zum 12MoFu ein Anstieg verzeichnen, der durch die erneute Operation der Partnerin verursacht sein könnte.

Bezüglich der dyadischen Funktionsfähigkeit (Abb. 2) zeigte der Mann eine gleichbleibend sehr hohe Zufriedenheit mit der Partnerschaft vom prä-Zeitpunkt zu 6 Monate nach post $(6 \mathrm{MoFu})$ mit einer leichten Reduktion zum $12 \mathrm{MoFu}$ und liegt damit zum prä-, post-Zeitpunkt und $6 \mathrm{MoFu}$ über den Werten der Männer insgesamt. Das gleiche Muster findet sich beim DC mit gleichbleibend hohen Zufriedenheitswerten zu allen Messzeitpunkten, die deutlich über den Werten der Männer insgesamt liegen. Bei der Frau fand sich ein Anstieg der Partnerschaftszufriedenheit vom prä- zum post-Zeitpunkt, die dann gleichbleibend hoch blieb und über den Werten der Frauen insgesamt lag. Dieser Anstieg vom prä- zum 
post-Zeitpunkt zeigte sich auch im DC, ebenfalls mit höheren Zufriedenheitswerten als bei den Frauen insgesamt. Beide Partner berichteten immer wieder von einer subjektiv erlebten Verbesserung der Kommunikation, die sich bei der Frau vom prä- zum post-Zeitpunkt darstellte. Beim Mann ist eher von einem «Deckeneffekt» auszugehen, da schon zum präZeitpunkt eine sehr hohe Zufriedenheit vorlag.

Zusammenfassend kann festgehalten werden, dass die hohe dyadische Zufriedenheit über den Zeitraum von über einem Jahr trotz physischer und psychischer Belastungen aufrechterhalten werden konnte (Präventionseffekt), wohingegen die Paare der Kontrollbedingung eine Abnahme der Zufriedenheit mit der Partnerschaft und dem DC (nur Frauen) zum post-Zeitpunkt und $6 \mathrm{MoFu}$ zeigten. Die psychischen Belastungen - vermutlich auch durch die medizinischen Interventionen verursacht - zeigen sich eher in der individuellen Funktionsfähigkeit der Frau mit erhöhten Werten bei der krebsbezogenen psychischen Belastung und vergleichbaren Progredienzangstwerten in Bezug zu den Frauen insgesamt.

Die spezifischen Bausteine des «Seite an Seite»-Programms in Bezug auf (1) die Aufklärung beider Partner über die Krebserkrankung und die medizinische Behandlung der Frau, (2) die Förderung der Fähigkeit beider Partner sich gegenseitig in der Auseinandersetzung mit der Erkrankung emotional und praktisch zu unterstützen und (3) die Vorbeugung von Problemen mit dem Körperbild und der Sexualität sollten flexibel - auf die Bedürfnisse des Paares angepasst - angewendet werden. Der Intervention liegt die Annahme der gemeinsamen Bewältigung der Erkrankung zugrunde. Der frühe Zeitpunkt der Intervention (möglichst direkt nach Diagnosestellung) soll späteren individuellen und dyadischen Problemen vorbeugen (universelle Prävention). Es kann allerdings sein, dass zu diesem Zeitpunkt bei dem Paar nur eine geringe Problemwahrnehmung vorliegt; die meisten Paare reagieren unabhängig von ihrer partnerschaftlichen Zufriedenheit mit einer kurzfristigen Erhöhung der partnerschaftlichen Kohäsion auf eine lebensbedrohliche
Erkrankung. Somit kann es sinnvoll sein, eine Intervention wie «Seite an Seite» auch zu einem späteren Zeitpunkt, wenn Paare bereits Probleme wahrnehmen oder von dauerhaften individuellen psychischen Beschwerden berichten, anzuwenden. Die Durchführung im Rehabilitationsbereich (nach Abschluss der akuten medizinischen Behandlung) ist bisher noch nicht ausreichend evaluiert. Allerdings sprechen Befunde dafür, dass es sinnvoll erscheint, insbesondere zu dem Zeitpunkt wenn der Alltag wieder zurückkehrt und Themen wie Sexualität vermutlich mehr an Bedeutung gewinnen, eine partnerschaftliche Intervention anzubieten, die eine Stabilisierung der Partnerschaft beabsichtigt (indizierte Prävention). Des Weiteren kann es sinnvoll sein Paare auszuwählen, die durch die Erkrankung ein hohes Maß an psychischer Belastung bei eher geringem Ausmaß an partnerschaftlicher Unterstützung und Partnerschaftszufriedenheit erleben [Martire et al., 2010]. Bei einer ausgeprägten psychischen Belastung durch die Krebserkrankung bzw. hoher Progredienzangst - wie es bei der Patientin der Fall war - scheint die «Seite an Seite»-Intervention nicht intensiv genug zu sein, insbesondere dann, wenn es zu massiven physischen Belastungen kommt. Hier könnte eine höherfrequente Intervention, die stärker auf die Bewältigung von Angst und psychischer Belastung bei Krebs zugeschnitten ist, hilfreicher sein [Herschbach et al., 2010].

Das hier vorgestellte partnerschaftliche Unterstützungsprogramm stellt einen vielversprechenden und - durch die geringe Frequenz - kosteneffektiven Ansatz zur Unterstützung von Paaren mit einer Brustkrebserkrankung dar. Weitere Evaluationen in anderen Settings, z.B. dem Rehabilitationsbereich oder zu anderen Zeitpunkten (nach Abschluss der medizinischen Behandlung), sind geplant.

\section{Disclosure Statement}

Es liegen keine bedeutsamen Interessenkonflikte vor.

\section{Literatur}

Bodenmann G: Verhaltenstherapie mit Paaren. Bern, Hans Huber, 2004

Heinrichs N, Zimmermann T: Bewältigung einer gynäkologischen Krebserkrankung in der Partnerschaft: Ein psycho-onkologisches Behandlungsprogramm für Paare. Göttingen, Hogrefe, 2008.

Herschbach P, Berg P, Waadt S, Duran G, Engst-

Hastreiter U, Henrich G, Book K, Dinkel A Group psychotherapy of dysfunctional fear of progression in patients with chronic arthritis or cancer. Psychother Psychosom 2010;79:31-38.

Kalaitzi C, Papadopoulos VP, Michas K, Vlasis K,

Skandalakis P, Fillippou D: Combined brief psychosexual intervention after mastectomy: effects on sexuality, body image, and psychological wellbeing. J Surg Oncol 2007;96:235-240.

Manne S, Badr H: Intimacy and relationship processes in couples' psychosocial adaptation to cancer. Cancer 2008;112(suppl 11):2541-2555.
Martire LM, Lustig AP, Schulz R, Miller GE, Helgeson VS: Is it beneficial to involve a family member? A meta-analysis of psychosocial interventions for chronic illness. Health Psychol 2004;23:599-611.

Martire LM, Schulz R, Helgeson VS, Small BJ, Sa-

ghafi EM: Review and meta-analysis of couple-oriented interventions for chronic illness. Ann Behav Med 2010;40:325-342

Osborn RL, Demoncada AC, Feuerstein M: Psycho-

social interventions for depression, anxiety, and quality of life in cancer survivors: meta-analyses. Int J Psychiatry Med 2006;36:13-34.

Robert Koch Institut, die Gesellschaft der epidemiologischen Krebsregister in Deutschland e.V. (Hrsg): Krebs in Deutschland 2005/2006. Häufigkeiten und Trends, ed 7. Berlin, 2010.
Zimmermann T, Heinrichs N: Psychosoziale Interventionen für Frauen mit Krebserkrankungen der Genitalorgane. Verhaltensther Verhaltensmed 2006;27:125-141.

Zimmermann T, Heinrichs N: «Seite an Seite». Eine gynäkologische Krebserkrankung in der Partnerschaft gemeinsam bewältigen - Ein Ratgeber für Paare. Göttingen, Hogrefe, 2008.

Zimmermann T, Heinrichs N: Auswirkungen einer psychoonkologischen Intervention für Paare auf die Sexualität bei einer Brustkrebserkrankung der Frau. Zeitschr Gesundheitspsych 2011;19:23-34.

Zimmermann T, Heinrichs N, Baucom DH: «Does

one size fit all?» Moderators in psychosocial interventions for breast cancer patients. A meta-analysis. Ann Behav Med 2007;34:225-239. 